\title{
Deletion of a Seminal Gene Cluster Reinforces a Crucial Role of SVS2 in Male Fertility
}

\author{
Miyuki Shindo ${ }^{1}\left(\mathbb{D}\right.$, Masafumi Inui ${ }^{2}{ }^{\circledR}$, Woojin Kang ${ }^{3}$, Moe Tamano ${ }^{4}$, Cai Tingwei ${ }^{5}$, \\ Shuji Takada ${ }^{4}$, Taku Hibino ${ }^{6}$, Manabu Yoshida ${ }^{7}$ (1) Kaoru Yoshida ${ }^{8}$, Hiroshi Okada ${ }^{9}$, \\ Teruaki Iwamoto ${ }^{10}$, Kenji Miyado ${ }^{3}$ (D) and Natsuko Kawano ${ }^{5, *}$
}

1 Division of Laboratory Animal Resources, National Research Institute for Child Health and Development, 2-10-1 Okura, Setagaya, Tokyo 157-8535, Japan

2 Laboratory of Animal Regeneration Systemology, Department of Life Sciences, School of Agriculture, Meiji University, 1-1-1 Higashimita, Kawasaki, Kanagawa 214-8571, Japan

3 Department of Reproductive Biology, National Research Institute for Child Health and Development, 2-10-1 Okura, Setagaya, Tokyo 157-8535, Japan

4 Department of Systems BioMedicine, National Research Institute for Child Health and Development, 2-10-1 Okura, Setagaya, Tokyo 157-8535, Japan

5 Laboratory of Regulatory Biology, Department of Life Sciences, School of Agriculture, Meiji University, 1-1-1 Higashimita, Kawasaki, Kanagawa 214-8571, Japan

6 Faculty of Education, Saitama University, 255 Shimo-Okubo, Sakura, Saitama, Saitama 338-8570, Japan

7 Misaki Marine Biological Station, Graduate School of Science, University of Tokyo, 1024 Koajiro, Miura, Kanagawa 238-0225, Japan

8 Faculty of Biomedical Engineering, Toin University of Yokohama, 1614 Kurogane, Aoba, Yokohama 225-8503, Japan

9 Department of Urology, Dokkyo Medical University Koshigaya Hospital, 2-1-50 Minamikoshigaya, Koshigaya, Saitama 343-8555, Japan

10 Division of Male Infertility, Center for Human Reproduction, Sanno Hospital, International University of Health and Welfare, 8-10-16 Akasaka, Minato, Tokyo 107-0052, Japan

* Correspondence: nkawano@meiji.ac.jp

Received: 30 July 2019; Accepted: 12 September 2019; Published: 14 September 2019

check for updates

\begin{abstract}
Multiple genes, whose functions or expression are overlapping, compensate for the loss of one gene. A gene cluster in the mouse genome encodes five seminal vesicle proteins (SVS2, SVS3, SVS4, SVS5, and SVS6). These proteins are produced by male rodents and function in formation of the copulatory plug following mating. SVS2 plays an essential role in the successful internal fertilization by protecting the sperm membrane against a uterine immune attack. We hypothesized that the four remaining seminal vesicle proteins (SVPs) of this gene cluster may partially/completely compensate for the deficiency of SVS2. For confirming our hypothesis, we generated mice lacking the entire SVP-encoding gene cluster and compared their fecundity with Svs2-deficient (Svs2 ${ }^{-/-}$) mice; that is, mice deficient in Svs2 alone. A single loxP site remained after the deletion of the Svs2 gene. Therefore, we inserted another loxP site by combining the CRISPR/Cas9 system with single-stranded oligodeoxynucleotides (ssODN). Male mice lacking the entire SVP-encoding gene cluster (Svs $2-6^{-/}$ mice) and thereby all five SVP proteins, generated by the deletion of 100kbp genomic DNA, showed low fecundity. However, the fecundity level was comparable with that from Svs $2^{-/-}$male mice. Our results demonstrate that SVS3, SVS4, SVS5, and SVS6 do not function in the protection of sperm against a uterine immune attack in the absence of SVS2. Thus, Svs2 is the critical gene in the SVP gene cluster.
\end{abstract}

Keywords: gene cluster; deletion; semen; seminal vesicle proteins; male fertility 


\section{Introduction}

A gene cluster is a set of multiple tandemly aligned genes that comprise homologous sequences and often encode proteins with similar functions. For example, the discovery of the homeobox (Hox) gene clusters revealed that body segments emerge and are multiplied via a mechanism conserved from insects to mammals, including humans [1]. On the contrary, a bacterial operon contains several genes and their harmonized regulatory system works under the control of a single promoter [2]. Gene clusters are believed to originate from a single ancestral gene, however the molecular basis underlying their formation and the biological significance of this process is largely unclear.

The semen, a mixture of fluids secreted from the male accessory reproductive organs, seminal vesicles, prostate gland, and bulbourethral glands, contributes to critical events during internal fertilization. Proteins contained in the semen (seminal proteins) rapidly diverge by adaptive evolution [3-6]. In primates, the semenogelins (SEMGs), which are seminal vesicle proteins (SVPs), constitute the principal structural components of semen coagulum in the female reproductive tract [7]. The gain of the gene coding SEMGII is correlated with species-specific semen coagulum and with levels of female promiscuity [8].

In rodents, the seminal vesicles secrete seven proteins, SVS1 to SVS7 [5]. As depicted in Figure 1A, Svs2 is localized in mice on chromosome 2 and the five genes (Svs3a, Svs3b, Svs4, Svs5, and Svs6) encoding components of seminal vesicle secretions (SVS3, SVS4, SVS5, and SVS6) are tandemly aligned with Svs2 in a gene cluster that is $\sim 100 \mathrm{kbp}$ long. The paralogous Svs $2-6$ genes are suggested to duplicate from a gene that originated from a whey acidic protein four-disulfide core (WFDC)-type proteinase gene ancestor, as well as from SEMGs $[9,10]$. Previously, we showed that SVS2 inhibits sperm fertility as well as human SEMGs [11]. SVS2 prevents cholesterol-efflux from the murine sperm membrane and incorporates free cholesterol into the sperm membrane [12]. These results show that SVS2 suppresses the release of sterols from the sperm plasma membrane and plays a key role in unlocking sperm capacitation. Moreover, SVS2 is required for survival of sperms in the female reproductive tract [13]. Based on their identical genomic loci and partial similarity of nucleotide sequences, the Svs2 to Svs6 genes in mice are thought to correspond to human SEMG1 and II genes (Figure 1A). Mouse SVS2 and SVS3 proteins share the SEMG domain with human SEMGI and II, albeit partially and only at the N-terminus (Figure 1B). SVS4, 5, and 6, with the SVS4_5_6 domain and without a SEMG domain, have a comparable effect as that of SVS2 and can compensate for the function of SVS2 [12]. The formation of a gene cluster often bestows similar, but possibly also distinct functions to genes such as those belonging to the Hox gene cluster. In this study, we generated mice lacking six serial (tandem) genes that encode SVPs to explore the role of a gene cluster in the formation of seminal vesicles and thereby male fertility. 

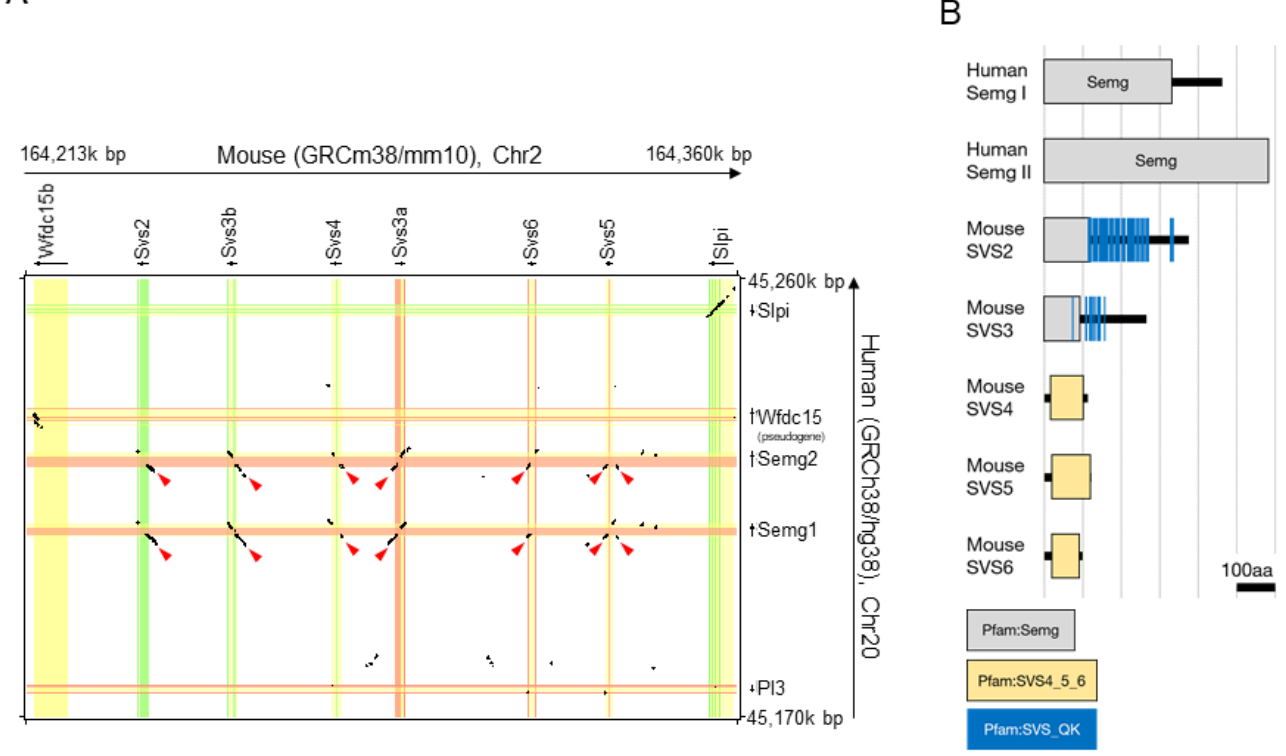

Figure 1. Comparative analysis of the mouse and human seminal proteins. (A) Dot-plot display of the mouse genome, including Svs2-6 ( $x$-axis), and the human genome, including SemgI and II (y-axis). Nucleotide positions are indicated for the mouse sequence on the $x$-axis, and sequence identity with the corresponding human sequence (50-100\%) is shown as black dots. Shading in orange and green indicates the locations of exons on the + and - strands, respectively. Yellow areas represent introns. Red arrow heads indicate that the human SemgI and II share gene similarities with mouse Svs2-6. (B) Domain architectures of the human SEMGI and II and the mouse SVS2-6. SEMGI and II, as well as SVS2 and 3 share a SEMG domain (gray box). SVS4-6 share other domains (yellow box).

\section{Results}

\subsection{Designing gRNA for Deletion of a Cluster of Svs2-6 Genes}

Svs2-deficient $\left(\mathrm{Svs}_{2}{ }^{--}\right)$mice were generated as previously described [13]. Here, we generated additional loxP-knockin mice that lacked six serially placed genes (Svs2, Svs3a, Svs3b, Svs4, Svs5, and Svs6) (Svs2-6 ${ }^{-/-}$mice) by combining the CRISPR/Cas9 system and ssODN (Figure 2A-C). The Cas9 mRNA, gRNAs, and ssODN were microinjected into pronuclear eggs fertilized with sperms derived from Svs2 $2^{-/}$mice in vitro (Figure 3A). We obtained 27 first generation (F0) pups from 99 injected embryos (Figure 3B). PCR amplification and sequencing revealed that 15 out of the 27 pups (56\%) carried mutations at the target locus and 10 out of 27 pups (37\%) tested positive for loxP cassette sequences. Six pups had a mutated loxP sequence and complete loxP sequences were detected in only four pups (15\%). As shown in Figure 4B, one F0 male mouse (\#711) had loxP knockin alleles, of which one was complete and another was a mutated loxP sequence. By mating this mouse with wild-type female mice, we generated second generation (F1) mice carrying a complete loxP sequence (Figure 3B). This F1 mice pool further comprised of two types of knockin mice: WT allele carrying a loxP cassette, and a Sus2-deficient allele carrying two loxP cassettes. The latter knockin mice, with cis-integrated-loxP, were further examined using PCR and sequenced with primers P1/P2 and P3/P4. 
A
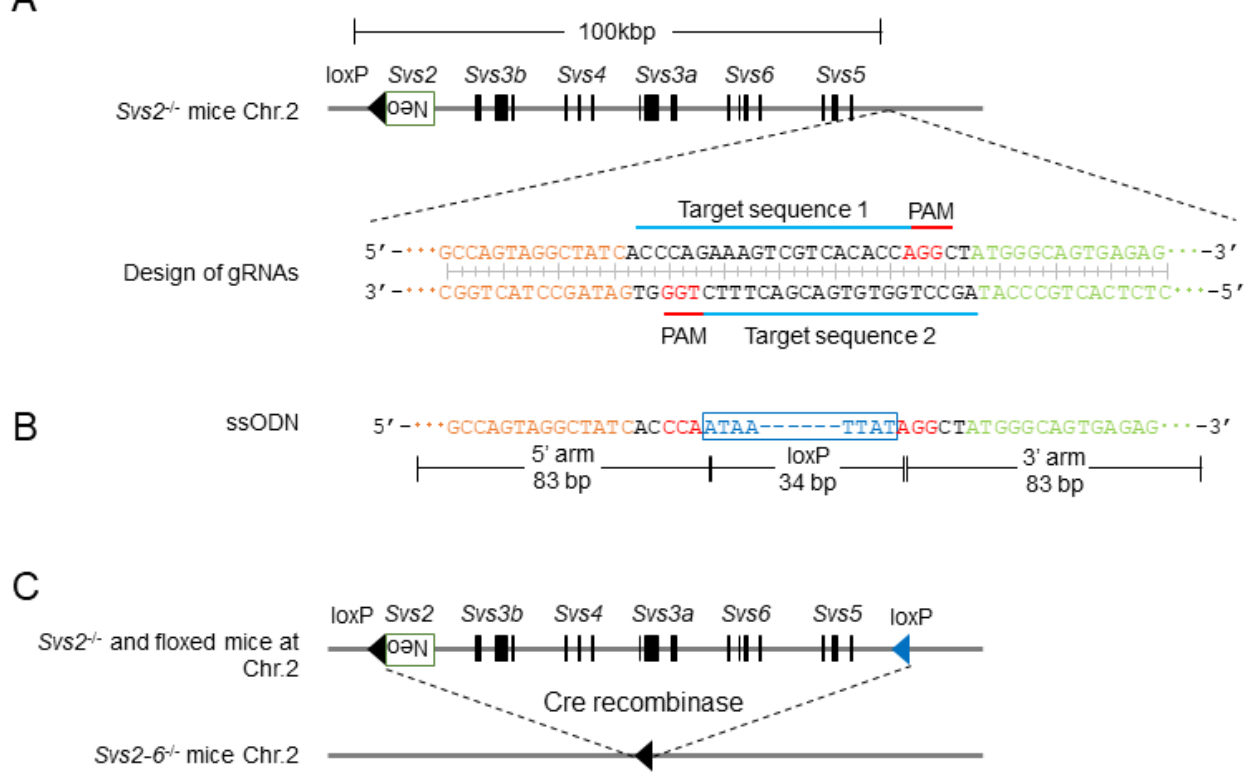

Figure 2. Genome editing strategy for knockin mice carrying a loxP cassette and knockout mice lacking five Svs genes. (A) Schematic representation of the Cas9-gRNA targeting sites. Two types of gRNAs were designed downstream of Svs5. Blue lines label the CRISPR target sequence. Red characters indicate protospacer adjacent motif (PAM) sequences. Orange and green characters indicate the upstream and the downstream region of the targeting site, respectively. (B) ssODN sequence for generating knockin mice. Knockin ssODN containing a loxP cassette and two 83 bp homology sequences. Blue characters indicate loxP sequence. Orange and green characters indicate homology arm with $5^{\prime}$ and $3^{\prime}$ of gRNA targeting sequence, respectively. (C) Generation of mice lacking Svs2-6 (almost $100 \mathrm{kbp}$ ) by a cre-recombination system.

A
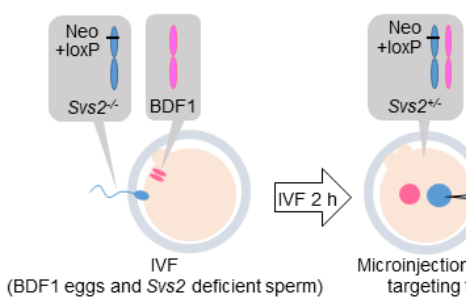

(BDF1 eggs and Svs2 deficient sperm)

Microinjection of Crispr/Cas9 and ssODN

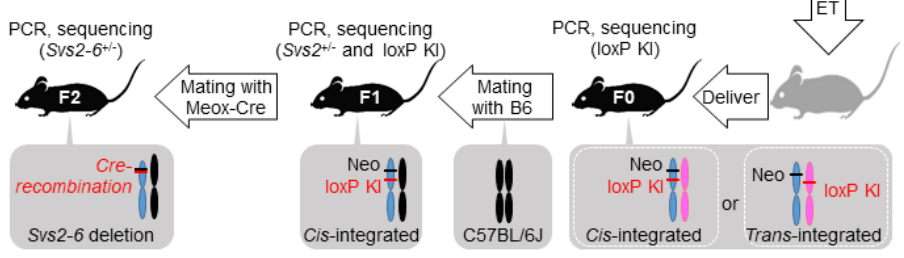

PCR, sequencing
(Svs2+/- and loxP KI)

argeting the downstream of Svs5

B

\begin{tabular}{|c|c|}
\hline Operation & Number of embryo \\
\hline Embryos injected & 99 \\
\hline Pups delivered & 33 \\
\hline Genotyping & 27 \\
\hline Mutation & $15(15 / 27=56 \%)$ \\
\hline $\mathrm{Kl}$ & $10(10 / 27=37 \%)$ \\
\hline Complete loxP & $4(4 / 27=15 \%)$ \\
\hline Cis-integrated loxP & $1(1 / 27=4 \%)$ \\
\hline
\end{tabular}

Figure 3. Experimental scheme for generating Svs2-6-- mice. (A) Co-injection of gRNAs, Cas9 mRNA, and ssODN into fertilized BDF1 eggs with Svs2-deficient sperm. The loxP cassette should be cis-integrated with blue chromosomes carrying Neo-loxP from Svs $2^{--}$mice. (B) Summary of the efficiency in CRISPR/Cas9 and ssODN induced mutations and loxP-knockin mice. 
A Svs2/- mice Chr.2 floxP Kl

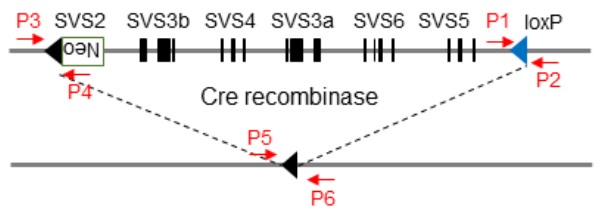

\begin{tabular}{|c|l|}
\hline Primers & Sequence ('5 - '3) \\
\hline P1 & GTGAAGACCAAAGGCTGAGG \\
\hline P2 & AGAGGGCCCATCCTGTTACT \\
\hline P3 & CAAACGTGGGGACTAAGCAT \\
\hline P4 & ATCTGGACGAAGAGCATCAG \\
\hline P5 & CATGGGGCTITTGTTGAT \\
\hline P6 & AGAGGGCCCATCCTGTTACT \\
\hline Cre_F & GCATTACCGGTCGATGCAACGAGTGATGAG \\
\hline Cre_R & GAGTGAACGAACCTGGTCGAAATCAGTGCG \\
\hline
\end{tabular}

B

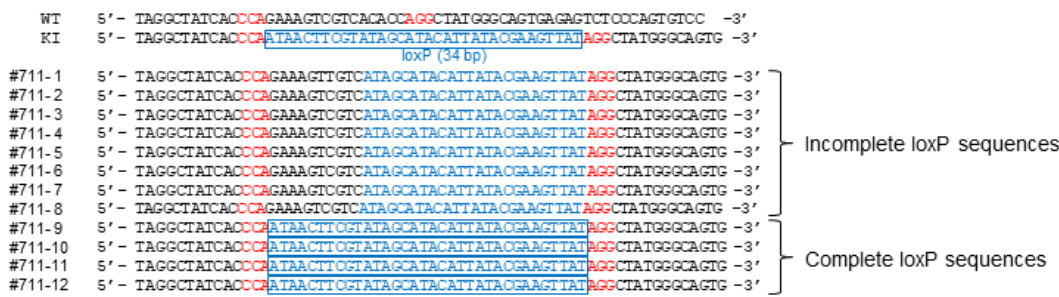

C

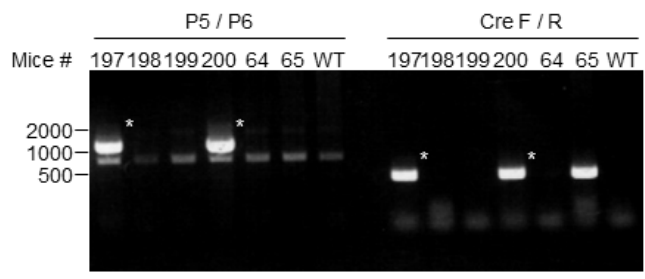

D

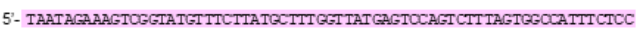
AGCT CAAAAATTGATGTTTCCATGGTGGTATTGGAATTGGAAACTAATCCOCTIGCOCOOGTGAT CITITEACTCTEACTGTCAGTITCTAAA GACOCACTAGAAAAACAGTTGTAAGTTATTTTATGGT I GACTITAOGTAACTCACA GOGCATCTGGCATAGTTGAGGGOCOCAATGCOCAGTACTAGT CAAC

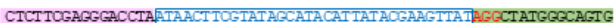
AGAGTCT OOCAGT GICCACTCA CACCAAGSCACA TAAATGAGGAGT ATGCCAATGACAAACACAA GAAACCACAT CAGTAGCAAGCAACAATCCTGAAA CAAAGT TOCTGGEACTAT COCTCACCA GAGC GCACTCATCACAGCTGAGTAACAGGATGGOCOCT CTCCAGGEA GCT CACTOGCOCACTACAACTT ITOCTOCATGGIGTCT COCAAAGTAGATAAAAAAAAAAAGTAGACA TACACCAACATT CAATCCA-3'

Figure 4. Validation of the $\mathrm{Svs}^{-{ }^{--}}$allele carrying a loxP-insertion using sequencing, PCR, and cre-recombination. (A) Primer sequences used in this study. (B) Sequence analysis of founder mouse (F0, \#711). Genomic DNA of the \#711 mouse was amplified by PCR (P1/P2) to detect loxP-insertion at the targeted locus. Genetic chimerism was observed in the \#711 mouse; both complete and incomplete loxP sequences were detected in the mouse. (C) PCR analysis of F2 pups (\#197-200, 64, 65) derived from crosses with F1 mice and Meox-cre Tg mice with primer sets P5/P6 as indicated in (A). PCR bands from Svs $2-6^{+--}$mice marked with asterisks display $1000 \mathrm{bp}$ and are cre-positive. (D) Sequences of PCR products with using P5/P6 in an F2 mouse. Blue characters indicated loxP sequences, and red means PAM sequence. Pink and green shading characters indicate $5^{\prime}$ upstream of Svs2 and $3^{\prime}$ downstream of Svs5, respectively.

\subsection{Generation of Sus $2-6^{-/-}$Mice}

To obtain third generation (F2) mice carrying the heterozygous $100 \mathrm{kbp}$ deletion, the F1 mice were mated with mice expressing a cre-recombinase (cre) under the control of a mesenchyme homeobox (Meox) gene (Meox-cre mice). Thus, we identified F2 mice carrying the $100 \mathrm{kbp}$ deletion $\left(\mathrm{Svs} 2-6^{+/-}\right)$by sequencing the combined sequences upstream of the Svs2 gene and downstream of Sus5 gene using primers P5/P6 and Cre_F/Cre_R (Figure 4C,D).

To confirm the generation of mice lacking SVPs (SVS2, SVS3, SVS4, SVS5, and SVS6), we performed SDS polyacrylamide gel electrophoresis (SDS-PAGE) using lysates of seminal vesicle secretions collected from Svs2- $6^{-/}$male mice. The polyacrylamide gels were stained with coomassie brilliant blue (Figure 5A). The resolved proteins were comparably expressed in the seminal vesicle secretions collected from wild-type (Svs2-6 ${ }^{+/+}$) and heterozygote $\left(\operatorname{Svs} 2-6^{+-}\right)$male mice. They were not detected in the seminal vesicle secretions collected from $S v s 2-6^{-/}$male mice. Since the two genes that encode SVS1 and SVS7 are localized on chromosomes separated from a gene cluster encoding SVS2 to SVS6, we assumed that the expression of these two proteins would remain unaffected. Unexpectedly, the expression of SVS1 protein was reduced and unidentified proteins were detected in Svs2- $6^{-/}$male mice, compared with Svs2- $6^{+/+}$and Svs2- $6^{+/-}$mice. In contrast, the expression of SVS7 was unaffected in Svs2-6 $6^{-/-}$male mice. 
A

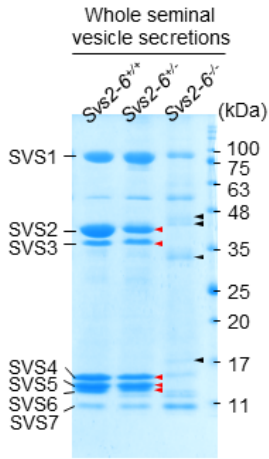

C

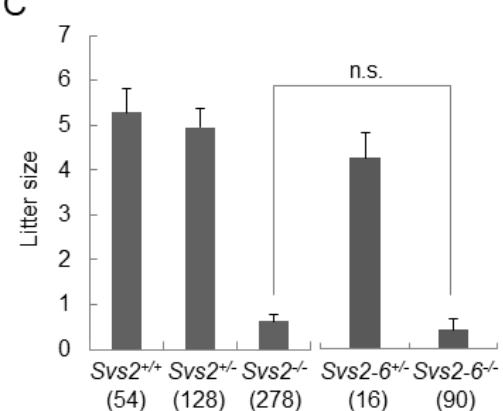

B

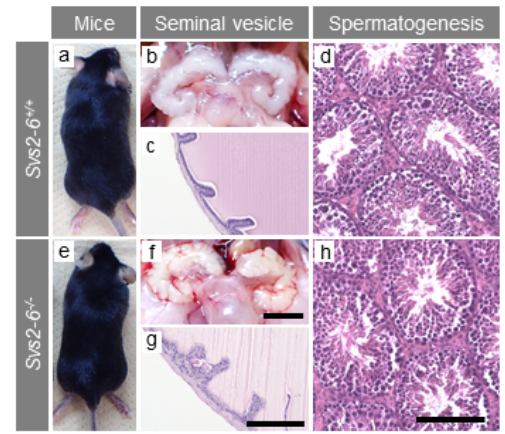

D

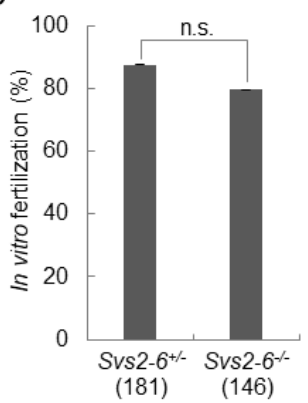

E

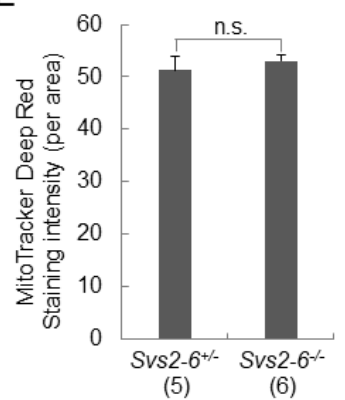

Figure 5. Reproductive phenotypes of $S v s 2-6^{-/}$male mice. (A) CBB staining of the total inner fluid of seminal vesicle isolated from male mice. Red arrow heads: Proteins that disappeared/not detected in Svs2- $6^{-/-}$male mice. Black arrow heads: Unknown proteins found in Svs2-6 $6^{-/-}$male mice. (B) Representative pictures of 8-week-old Svs2-6 ${ }^{+/+}$(a) and Svs2-6 $6^{-/-}$(e) mice. Appearance of seminal vesicles collected from 8 -week-old mice ( $\mathrm{b}$ and f). Scale bar, $5 \mathrm{~mm}$. Hematoxylin and eosin (H\&E) stained sections of seminal vesicle tissue from 8-week-old mice (c and g). Scale bar, $100 \mu \mathrm{m}$. Testicular sections stained with H\&E ( $\mathrm{d}$ and h). Scale bar, $100 \mu \mathrm{m}$. (C) Fecundity of male mice. Parentheses, numbers of male mice examined. (D) The rate of fertilization of eggs with epididymal sperm collected from Svs2-6 $6^{+/-}$and Svs2-6- ${ }^{-/}$mice. Values indicate mean \pm SEM of triplicate experiments. Numbers in parentheses indicate the number of eggs examined. (E) Fluorescence intensity of mitochondrial staining

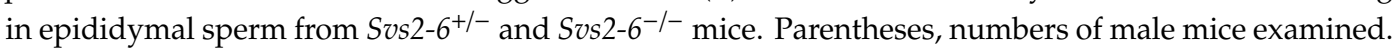

Svs $2-6^{-/-}$mice were born healthy and grew normally (Figure 5B). Despite being deficient in Svs2, Svs3a, Svs3b, Svs4, Svs5, and Svs6, the seminal vesicles were formed normally. This result suggests that a specific gene cluster encoding SVPs is not essential for the growth and differentiation of seminal vesicles.

\subsection{Male Fertility of Svs2- $6^{-/}$Mice}

Our previous report showed that the fertility of $\mathrm{Svs}^{-/-}$male mice was strongly reduced [13]. The fertility of $\mathrm{Svs} 2-6^{-/-}$male mice was also reduced but was comparable with mice lacking a single Svs2 gene $(0.43+/-0.14$ and $0.63+/-0.13$, respectively; $p=0.420)$ (Figure $5 C$ ). Similar to Svs $2^{-/}$male mice, no copulatory plugs were formed inside the vagina of female mice that mated with Svs2- $6^{-/}$male mice, in spite of observed mating behavior and normal production of sperms in these mice (Figure $5 B$ ). In vitro fertilization (IVF) and a mitochondrial activity assay showed that the epididymal sperms of Svs2- $6^{-/}$male mice were comparable to those of Svs2- $6^{+/-}$male mice (Figure 5D-E). It is known that hormonal condition is important for acquisition and maintenance of male fertility. Although we previously measured testosterone concentration in the serum of male mice [13,14], there were no significant differences due to individual variability but not genotypes. Therefore, we removed the results of hormonal measurements in this study. Otherwise, sex behavior, spermatogenesis, and sperm quality in Svs $2-6^{-/-}$mice are comparable to that of $S v s 2-6^{+/+}$male mice, indicating that hormonal concentrations are normal in Svs2-6- ${ }^{-/-}$mice. 
On the basis of this, we concluded that the SVS3-6 proteins play no additive roles, at least in male fertility, and the formation of seminal vesicles. Thus, SVS2 is the sole key factor for regulating male fertility through seminal vesicle secretions.

\section{Discussion}

To gain a comprehensive understanding of the function of the Svs gene family, we generated Svs $2-6^{-1-}$ mice lacking the entire Svs2-6 gene locus. Although epididymal sperms collected from Svs $2-6^{--}$mice were normal in morphology and quality, their fertility was quite low in the female reproductive tract (Figure 5C-E). Moreover, we observed no significant difference between the Svs $2-6^{-/}$ mice and the Svs $2^{-/}$male mice generated in an earlier study [13], except that the fecundity with sperms from the Svs2-6 $6^{-/}$male mice tended to reduce slightly compared with those from the Svs $2^{-/}$ male mice (Figure 5C). This result is consistent with our previous study that the ganglioside GM1, functional as a sperm receptor, interacts with SVS2 at a higher level than those of SVS3 and SVS4 [12]. Moreover, SVS2 proteins aid epididymal sperms achieve uterine survival without SVS3-6 [13]. Ramm et al. [5] used comparative proteomic techniques and reported that SVS2 correlates positively with sperm competition levels across species. The results of the current study in combination with literature evidences indicate the pivotal role of SVS2 in uterine sperm survival in seminal plasma. They point out that SVS3-6 proteins are unable to compensate for SVS2 deficiency in internal fertilization.

In natural mating, SVS2 is degraded in various length in the female reproductive tract [11]. Similarly, SEMG is degraded by the serine protease termed prostate-specific antigen (PSA) secreted from the prostate in humans [15]. From the fragmentation of SVPs in humans and mice, we hypothesize that SVS2 and SEMGI/II contain a functionally conserved domain with multiple or short sequences. Actually, SEMGI and II include repeated units and work as a sperm motility inhibitor and bactericidal agent after their degradation [16-18]. Although the mechanism of acquisition of SVS2 function is still unclear, the domain analysis reveals unique repetitive domains, SVS_glutamine and lysine (QK), which are repeated 17 times in the SVS2 sequence (Figure 1A). A single SVS_QK domain consists of 12 amino acids including basic amino acids $\mathrm{K}$, and is detected only in SVPs derived from rats and mice in mammalian species. Since none of the SVS4, 5, and 6 have this domain, we assumed that the SVS_QK domain could be evolved after expansion of the Svs gene cluster. Although the function of the SVS_QK domain is unclear, the constant repeat of basic amino acids may enable immunological tolerance in the female reproductive tract and/or sperm protection against female immune system.

The Svs gene cluster emerged by a tandem duplication in recent evolution [5]. The genes consist of three exons, and the nucleotide sequences of the Svs2-6 genes are highly similar with the human SemgI and II at the first exons and their upstream regions that contain predicted promoter regions (Figure 1A). Differences are observed in the second exon which encodes almost all of the secreted protein that serves in formation of a semen gel or clot (copulatory plug). The amino acid sequences of the secreted proteins encoded by Svs2-6 genes are diverse and this diversification can be explained by the rapid evolution of the Svs gene family, which corresponds to adaptation against species-specific mating behavior and/or speciation [8,19]. In a similar case, several gene families such as genes encoding the major histocompatibility complex (MHC), antimicrobial peptides, and immunoglobulin typically have several paralogs and pseudogenized duplicated genes [20-23]. A group of MHC class I-like molecules, known as NKG2D ligands, are prime targets for immune evasion strategies deployed by many viruses [24]. An ancestral NKG2DL gene located in the MHC region underwent duplication, subsequently producing a MIC gene and MILL gene [25]. These two genes have been retained in the marsupial opossum, however the MILL gene was lost in humans, while the MIC gene was lost in rodents. The species-specific evolution of the NKG2DL gene family causes infection of the species-specific pathogen. From this immunological viewpoint, SVS2 proteins in mice mainly function in protection of sperms against female immune response in the uterus. The SVS3-6 proteins or further new duplications may serve as an adaptor response against the evolution of female spermicide and sperm receptors against SVS2. 
Eukaryotic genes are considered to be independently expressed under the control of their own promoters and cis-regulatory elements. As mentioned above, genes co-habited on a chromatin domain or genomic neighborhood are co-expressed frequently, but these co-expressed genes are often free from functional partnership within the same biological phenomena [26]. Although the mechanisms for partitioning the genome into clusters of co-expressed genes are poorly understood, their conservation of clustering across species suggests that this organization did not arise randomly but selectively. Therefore, the perturbation of such an organization may prove deleterious for the continued existence of a species. In the context of human diseases, dysregulation of gene expression across genomic neighborhoods causes highly pleiotropic diseases, including genomic instability disorders, global covalent histone modifications, and DNA methylation. In case of seminal vesicle secretions in Svs $2-6^{-/}$ mice, we observed an increased expression of unknown proteins, which could not be detected in Svs $2-6^{+/-}$mice (Figure 5A). This implied that the deletion of a seminal gene cluster in mice could disturb genomic neighborhoods, presumably leading to short-time speciation in vitro.

In conclusion, we succeeded in generating Svs2-6 $6^{--}$mice with a $100 \mathrm{kbp}$ genomic deletion by combining CRISPR/Cas9 system with ssODN. We confirmed that SVS2 plays the major role in male fertility by functioning as a component of the murine seminal plasma. Present diagnosis of male infertility is made by sperm number and quality but seminal plasma proteins surrounding the sperm, because the importance of seminal plasma proteins in internal fertilization is ignored in humans. Our results raise the possibility that lack of a seminal protein, i.e., human SEMGI/II, may cause male infertility in humans. In near future, the evaluation of seminal plasma proteins (quantity and quality) may be added to the diagnosis check list of infertile patients.

\section{Materials and Methods}

\subsection{Production of Knockout Mice}

Svs2-deficient $\left(\mathrm{Svs}^{-/-}\right)$mice were generated as previously described [13]. Here, we produced mice lacking six serial genes ( $S v s 2, S v s 3 a, S v s 3 b, S v s 4, S v s 5$, and $S v s 6)$ (Svs2- $6^{-/-}$mice) by combining the CRISPR/Cas9 system with single-stranded oligodeoxynucleotides (ssODN). Software tools (crispr.genome-engineering.org), which predict unique target sites that recognize the downstream of Svs5 throughout the mouse genome, were used for designing gRNAs (Figure 2A). The 19 base sequences were cloned into a modified gRNA cloning vector (Addgene plasmid ID 41824, Watertown, MA, USA) and served as templates for in vitro transcription using mMassagemMachine T7 kit (Thermo Fisher Scientific Inc., Waltham, MA, USA) as previously described [27]. ssODN included homology arms (83 bp) to link the double strand break of the original mouse DNA, and a 34 bp loxP cassette sequence was purchased from Integrated DNA Technologies Inc. (Coralville, Iowa, USA) (Figure 2B).

Eggs were collected from the BDF1 strain of superovulated female mice (Figure 3A). ssODN with gRNAs and Cas9 mRNA prepared by hCas9 expressing vector (Addgene, plasmid ID 41815) were microinjected into pronuclear eggs fertilized with the sperms derived from Svs2 $2^{-/}$mice. Embryos that were developed to the two-cell stage were transferred to pseudo-pregnant ICR female mice. F0 generation mice were genotyped by PCR amplification using primers P1/P2 and the sequencing of inserted loxP (Figure 4A). F1 mice (Svs2 ${ }^{+/-}$, loxP-KI) produced by crossing F0 with C57BL/6, were genotyped by PCR and sequenced by using primers $\mathrm{P} 1 / \mathrm{P} 2$ for detecting the inserted loxP and primers P3/P4 for confirming Svs2 deficiency (Figure 4A). F2 mice (Svs2- $6^{+-}$) generated by crossing F1 with B6; 129S4-Meox $2<\operatorname{tm} 1$ (cre)Sor $>/ J$ (Meox-cre Tg mice) [28], were genotyped by PCR and sequenced using primers P5/P6 and Cre_F/R (Figure 4A). We defined the $S v s 2-6^{-/-}$mice as those bearing a deletion of $100 \mathrm{kbp}$ including Svs2, Svs3a, Svs4, Svs3b, Svs6, and Svs5 on chromosome 2.

All mice were housed in specific pathogen-free controlled conditions. Food and water were available ad libitum. The procedures for performing animal experiments were approved by The Institutional Animal Care and Use Committee of the National Research Institute for Child Health and Development (1/April/2004). 


\subsection{Preparation of Seminal Vesicle Secretions}

Seminal vesicles were isolated from 8- to 20-week-old male mice, and the fluids extracted from the seminal vesicles were dissolved completely in $1 \mathrm{~mL}$ of $8 \mathrm{M}$ urea. Each sample was separated by SDS-PAGE and stained with coomassie brilliant blue (CBB) as previously described [11]. All experiments were performed with the approval of the Animal Care Committee of the Meiji University (IACUC15-0014).

\subsection{Male Fertility In Vivo and In Vitro}

To evaluate male fertility in vivo, the number of pups delivered by 8- to 16-week-old female mice were recorded after a 2-week mating period, during which two female mice were housed with a single 8- to 16-week-old male mouse.

For in vitro fertilization (IVF), eggs were collected from superovulated C57BL/6J female mice (8-12 weeks old) 14-16 h after human chorionic gonadotropin (hCG) injection [13]. The sperm collected from the epididymides of 12- to 24-week-old male mice were capacitated by incubating in HTF medium for 2 $\mathrm{h}$ before insemination. The final concentration of the sperm added to the eggs was $1.5 \times 10^{5} \mathrm{sperm} / \mathrm{mL}$.

Sperm mitochondrial activity in vitro was evaluated by staining with MitoTracker Deep Red FM (Thermo Fisher Scientific Inc., Waltham, MA, USA) as described previously [29]. Epididymal sperms from male mice were incubated in medium containing $500 \mathrm{nM}$ MitoTracker Deep Red FM for $30 \mathrm{~min}$ at $37^{\circ} \mathrm{C}$ and observed using a confocal laser microscope (LSM510Meta: excitation at $633 \mathrm{~nm}$ and emission at $650 \mathrm{~nm})$.

Data are presented as mean values \pm standard error of the mean. The Student's t-test was used for statistical analysis; significance was assumed at $p<0.05$.

Author Contributions: N.K. designed the outline of this study. M.S., M.I., and K.M. designed the experimental strategy. M.T. and S.T. made the knockout mice and W.K. and C.T. maintained the mice. M.S., T.H. and N.K. performed experiments in mice and analyzed the data. M.S., K.M. and N.K. wrote and M.I., T.H., K.Y., M.Y., T.I. and H.O. edited the manuscript. All authors agreed to the final version of the manuscript.

Funding: This study was supported by JSPS KAKENHI Grant Numbers 19K06474, 17K18164 and AMED under Grant Number 17930555.

Conflicts of Interest: The authors have no conflict of interest to declare.

\section{References}

1. Ferrier, D.E.; Holland, P.W. Ancient origin of the Hox gene cluster. Nat. Rev. Genet. 2001, 2, 33-38. [CrossRef] [PubMed]

2. Lawrence, J. Selfish operons: The evolutionary impact of gene clustering in prokaryotes and eukaryotes. Curr. Opin. Genet. Dev. 1999, 9, 642-648. [CrossRef]

3. Swanson, W.J.; Vacquier, V.D. The rapid evolution of reproductive proteins. Nat. Rev. Genet. 2002, 3, 137-144. [CrossRef] [PubMed]

4. Karn, R.C.; Clark, N.L.; Nguyen, E.D.; Swanson, W.J. Adaptive Evolution in Rodent Seminal Vesicle Secretion Proteins. Mol. Biol. Evol. 2008, 25, 2301-2310. [CrossRef] [PubMed]

5. Ramm, S.A.; McDonald, L.; Hurst, J.L.; Beynon, R.J.; Stockley, P. Comparative Proteomics Reveals Evidence for Evolutionary Diversification of Rodent Seminal Fluid and Its Functional Significance in Sperm Competition. Mol. Biol. Evol. 2009, 26, 189-198. [CrossRef] [PubMed]

6. Claw, K.G.; George, R.D.; MacCoss, M.J.; Swanson, W.J. Quantitative evolutionary proteomics of seminal fluid from primates with different mating systems. BMC Genom. 2018, 19, 488. [CrossRef] [PubMed]

7. Robert, M.; Gagnon, C. Semenogelin I: A coagulum forming, multifunctional seminal vesicle protein. Cell. Mol. Life Sci. CMLS 1999, 55, 944-960. [CrossRef] [PubMed]

8. Dorus, S.; Evans, P.D.; Wyckoff, G.J.; Choi, S.S.; Lahn, B.T. Rate of molecular evolution of the seminal protein gene SEMG2 correlates with levels of female promiscuity. Nat. Genet. 2004, 36, 1326-1329. [CrossRef]

9. Clauss, A.; Lilja, H.; Lundwall, A. A locus on human chromosome 20 contains several genes expressing protease inhibitor domains with homology to whey acidic protein. Biochem. J. 2002, 368, 233-242. [CrossRef] 
10. Clauss, A.; Lilja, H.; Lundwall, A. The evolution of a genetic locus encoding small serine proteinase inhibitors. Biochem. Biophys. Res. Commun. 2005, 333, 383-389. [CrossRef]

11. Kawano, N.; Yoshida, M. Semen-coagulating protein, SVS2, in mouse seminal plasma controls sperm fertility. Biol. Reprod. 2007, 76, 353-361. [CrossRef] [PubMed]

12. Araki, N.; Trencsenyi, G.; Krasznai, Z.T.; Nizsaloczki, E.; Sakamoto, A.; Kawano, N.; Miyado, K.; Yoshida, K.; Yoshida, M. Seminal Vesicle Secretion 2 Acts as a Protectant of Sperm Sterols and Prevents Ectopic Sperm Capacitation in Mice. Biol. Reprod. 2015, 92, 8. [CrossRef] [PubMed]

13. Kawano, N.; Araki, N.; Yoshida, K.; Hibino, T.; Ohnami, N.; Makino, M.; Kanai, S.; Hasuwa, H.; Yoshida, M.; Miyado, K.; et al. Seminal vesicle protein SVS2 is required for sperm survival in the uterus. Proc. Natl. Acad. Sci. USA 2014, 141, 4145-4150. [CrossRef] [PubMed]

14. Miyado, M.; Yoshida, K.; Miyado, K.; Katsumi, M.; Saito, K.; Nakamura, S.; Ogata, T.; Fukami, M. Knockout of Murine Mamld1 Impairs Testicular Growth and Daily Sperm Production but Permits Normal Postnatal Androgen Production and Fertility. Int. J. Mol. Sci. 2017, 18, 1300. [CrossRef] [PubMed]

15. Amelar, R.D. Coagulation, liquefaction and viscosity of human semen. J. Urol. 1962, 87, 187-190. [CrossRef]

16. Jensen-Seaman, M.I.; Li, W.-H. Evolution of the hominoid semenogelin genes, the major proteins of ejaculated semen. J. Mol. Evol. 2003, 57, 261-270. [CrossRef] [PubMed]

17. Edstrom, A.M.L.; Malm, J.; Frohm, B.; Martellini, J.A.; Giwercman, A.; Morgelin, M.; Cole, A.M.; Sorensen, O.E. The major bactericidal activity of human seminal plasma is zinc-dependent and derived from fragmentation of the semenogelins. J. Immunol. 2008, 181, 3413-3421. [CrossRef]

18. Iwamoto, T.; Tsang, A.; Luterman, M.; Dickson, J.; Delamirande, E.; Okuno, M.; Mohri, H.; Gagnon, C. Purification and Characterization of a Sperm Motility-Dynein Atpase Inhibitor from Boar Seminal Plasma. Mol. Reprod. Dev. 1992, 31, 55-62. [CrossRef]

19. Lundwall, A. The cloning of a rapidly evolving seminal-vesicle-transcribed gene encoding the major clot-forming protein of mouse semen. Eur. J. Biochem. 1996, 235, 424-430. [CrossRef]

20. Hughes, A.L.; Yeager, M. Natural selection at major histocompatibility complex loci of vertebrates. Annu. Rev. Genet. 1998, 32, 415-435. [CrossRef]

21. Ota, Y.; Tamegai, H.; Kudo, F.; Kuriki, H.; Koike-Takeshita, A.; EOUCHI, T.; Kakinuma, K. Butirosin-biosynthetic gene cluster from Bacillus circulans. J. Antibiot. 2000, 53, 1158-1167. [CrossRef] [PubMed]

22. Semple, C.A.M.; Rolfe, M.; Dorin, J.R. Duplication and selection in the evolution of primate beta-defensin genes. Genome. Biol. 2003, 4. [CrossRef]

23. Fawcett, J.; Innan, H. Neutral and non-neutral evolution of duplicated genes with gene conversion. Genes 2011, 2, 191-209. [CrossRef] [PubMed]

24. Jonjić, S.; Polić, B.; Krmpotić, A. Viral inhibitors of NKG2D ligands: Friends or foes of immune surveillance? Eur. J. Immunol. 2008, 38, 2952-2956. [CrossRef] [PubMed]

25. Kondo, M.; Maruoka, T.; Otsuka, N.; Kasamatsu, J.; Fugo, K.; Hanzawa, N.; Kasahara, M. Comparative genomic analysis of mammalian NKG2D ligand family genes provides insights into their origin and evolution. Immunogenetics 2010, 62, 441-450. [CrossRef] [PubMed]

26. Elizondo, L.I.; Jafar-Nejad, P.; Clewing, J.M.; Boerkoel, C.F. Gene clusters, molecular evolution and disease: A speculation. Curr. Genom. 2009, 10, 64-75. [CrossRef] [PubMed]

27. Inui, M.; Mokuda, S.; Sato, T.; Tamano, M.; Takada, S.; Asahara, H. Dissecting the roles of miR-140 and its host gene. Nat. Cell. Biol. 2018, 20, 516. [CrossRef]

28. Tallquist, M.D.; Soriano, P. Epiblast-restricted Cre expression in MORE mice: A tool to distinguish embryonic vs. extra-embryonic gene function. Genesis 2000, 26, 113-115. [CrossRef]

29. Iwai, M.; Harada, Y.; Miyabayashi, R.; Kang, W.; Nakamura, A.; Kawano, N.; Miyamoto, Y.; Yamada, M.; Hamatani, T.; Miyado, M.; et al. Chemotactic behavior of egg mitochondria in response to sperm fusion in mice. Heliyon 2018, 4, e00944. [CrossRef]

(C) 2019 by the authors. Licensee MDPI, Basel, Switzerland. This article is an open access article distributed under the terms and conditions of the Creative Commons Attribution (CC BY) license (http://creativecommons.org/licenses/by/4.0/). 